\title{
Superconducting Analogue of Optical Plasmonic Waveguides
}

\author{
A. Tsiatmas, V. A. Fedotov and N. I. Zheludev \\ Optoelectronics Research Centre and Centre for Photonic Metamaterials, University of Southampton, SO17 1BJ, UK
}

We demonstrate a direct analogy between electromagnetic properties of superconductors at frequencies up to 6 $\mathrm{THz}$ (superconducting gap) and plasmonic metals in the optical part of the spectrum. We also identify the existence of a surface bound mode in superconducting waveguide structures, "superconducting plasmon", that closely connected to surface plasmon polaritons in the noble metals. This is a peculiar low-frequency, low-loss mode that can be guided for tens of centimetres and confined on the scale of just few tens of nanometres, demonstrating an incredible application potential.

The plasmonic regime in the noble metals manifests itself just below the plasma frequency, when the free electrons driven by an electromagnetic wave oscillate so rapidly that almost no collisions happen during one period of oscillations. Correspondingly oscillations of all the electrons become coherent and their acceleration is determined by the strength of the incident field and kinetic inductance of the electrons. Intriguingly, similar situation is inherent to the superconducting state from substantially lower frequencies, where the absence of electron scattering and dominant kinetic inductance result from the formation of the Cooper pairs. This is also reflected by the negligible imaginary part of the complex dielectric constant compare to the real part, which is negative. Therefore we argue that coupled excitations much like optical plasmons in metals should exist in the superconductors at frequencies that fall in the superconducting gap.

Our previous experiments on extraordinary transmission through perforated superconducting films have indirectly shown the existence of the superconducting plasmons, which were held responsible for the enhancement of the effect upon superconducting transition [1]. Here we present the first direct evidence of highly localised plasmonic TM-modes supported by high- $\mathrm{T}_{\mathrm{c}}$ superconducting gap- and groove-waveguides of sub-micrometre lateral dimensions. Figure 1 shows the effective refractive index for the $100 \mathrm{GHz}$ gap-plasmon mode propagating in the air gap between two parallel YBCO layers (see inset to Fig. 1(a)) as a function of their separation and temperature. Remarkably, not only a mode with such extreme 1D-confinement can be sustained, but its propagation distance extends for many wavelengths adding up to several centimetres.
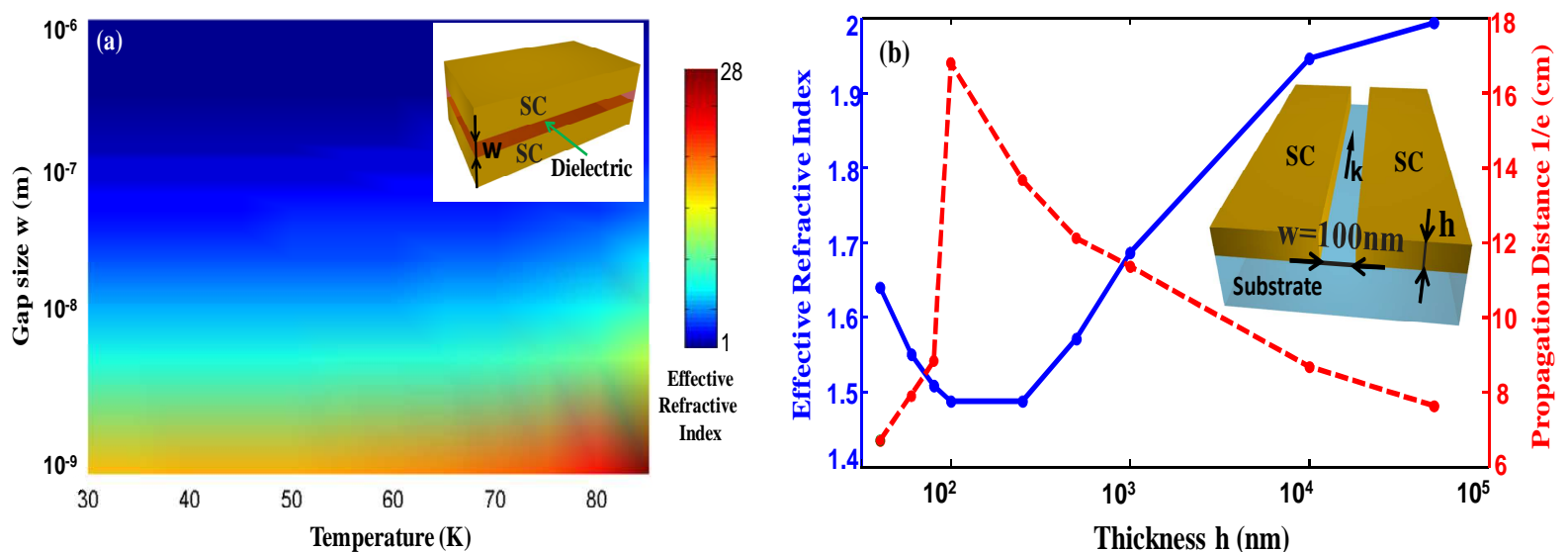

Fig. 1 (a) Dependence of the effective refractive index on temperature and gap size for an YBCO gap waveguide. Inset shows a representation of the structure. (b) Propagation distance and effective refractive index as a function of the gap width for an YBCO groove waveguide. Inset shows a representation of the structure.

In the case of a superconducting groove-waveguide (see inset to Fig. 1(b)) nanoscale lateral confinement of the plasmonic mode can be achieved in two dimensions. For example, a 100nm wide groove made in $500 \mathrm{~nm}$ thick YBCO film at $30 \mathrm{~K}$ contains about $80 \%$ of the energy of the superconducting plasmon propagating inside the groove at $100 \mathrm{GHz}$. In this particular configuration the mode propagates for distance of more than $14 \mathrm{~cm}$ with the effective refractive index of 1.5 (see Fig. 1(b)). The cross-section of such a superconducting plasmonic waveguide is about $2 \times 10^{7}$ times smaller than that of the conventional millimetrewave frequency waveguides. Importantly, given that at superconducting regime the dielectric permittivity of the material has very weak frequency dependence, the described waveguide modes will exhibit no dispersion paving the way towards dispersion-less plasmonics.

\section{References}

[1] A. Tsiatmas, A. R. Buckingham, V. A. Fedotov, S. Wang, Y. Chen, P. A. J. de Groot and N.I. Zheludev, Appl. Phys. Lett. 97 (1), $111106(2010)$ 